\title{
Growth of Meromorphic Function Sharing Functions and Some Uniqueness Problems
}

\author{
Jianming Qi, ${ }^{1,2}$ Fanning Meng, ${ }^{3}$ and Wenjun Yuan ${ }^{3}$ \\ ${ }^{1}$ Department of Mathematics and Physics, Shanghai Dianji University, Shanghai 201306, China \\ ${ }^{2}$ Department of Physics and Mathematics, University of Eastern Finland, 80101 Joensuu, Finland \\ ${ }^{3}$ School of Mathematics and Information Science, Guangzhou University, Guangzhou 510006, China \\ Correspondence should be addressed to Fanning Meng; mfnfdbx@163.com and Wenjun Yuan; wjyuan1957@126.com
}

Received 29 March 2016; Accepted 5 May 2016

Academic Editor: Kehe Zhu

Copyright (C) 2016 Jianming Qi et al. This is an open access article distributed under the Creative Commons Attribution License, which permits unrestricted use, distribution, and reproduction in any medium, provided the original work is properly cited.

Estimating the growth of meromorphic solutions has been an important topic of research in complex differential equations. In this paper, we devoted to considering uniqueness problems by estimating the growth of meromorphic functions. Further, some examples are given to show that the conclusions are meaningful.

\section{Introduction and Main Results}

Assuming that the reader is familiar with the notations and results on Nevanlinna theory [1] and the applications of normal family theory on estimating the growth of meromorphic functions (see [2-4]), it is an interesting attempt to consider the growth properties of meromorphic functions under the condition involved sharing value or some complex differential (or difference) equations (see [5-9]).

For a meromorphic function $f$, the order $\rho(f)$ and hyperorder $\sigma(f)$ of $f$ are defined as follows [1]:

$$
\begin{aligned}
\rho(f) & :=\limsup _{r \rightarrow \infty} \frac{\log T(r, f)}{\log r}, \\
\sigma(f) & :=\limsup _{r \rightarrow \infty} \frac{\log \log T(r, f)}{\log r} .
\end{aligned}
$$

Let $f(z)$ and $g(z)$ be two nonconstant meromorphic functions in the complex plane $\mathbb{C}$, and let $\alpha(z)$ be a meromorphic function or a finite complex number. If $g(z)-\alpha(z)=0$ whenever $f(z)-\alpha(z)=0$, we write $f(z)=\alpha(z) \Rightarrow g(z)=$ $\alpha(z)$. If $f(z)=\alpha(z) \Rightarrow g(z)=\alpha(z)$ and $g(z)=\alpha(z) \Rightarrow$ $f(z)=\alpha(z)$, we write $f(z)=\alpha(z) \Leftrightarrow g(z)=\alpha(z)$ and say that $f(z)$ and $g(z)$ share $\alpha(z)$ IM (ignoring multiplicity). If $g(z)-\alpha(z)=0$ whenever $f(z)-\alpha(z)=0$ and the multiplicity of the zero $z_{0}$ of $g-\alpha$ is greater than or equal to that of the zero $z_{0}$ of $f-\alpha$, then we denote this condition by $f(z)-\alpha(z)=0 \mapsto g(z)-\alpha(z)=0$. Let $R$ be a rational function which behaves asymptotically $c r^{\beta}$ as $r \rightarrow \infty$, where $c \neq 0, \beta$ are constants. The degree of $R$ at infinity is defined as $\operatorname{deg} R:=\operatorname{deg}_{\infty} R:=\max \{0, \beta\}$.

In the following, for a linear differential polynomial of $f$, we write

$$
L(f):=a_{k} f^{(k)}+a_{k-1} f^{(k-1)}+\cdots+a_{2} f^{\prime \prime}+a_{1} f^{\prime}+a_{0} f
$$

where $a_{1}, a_{2}, \ldots, a_{k}(\neq 0)$ are constants and $k \geq 2$ is an integer.

In 1986, Jank et al. [10] proved that, for an entire function $f$, if $f$ and $f^{\prime}$ share a finite nonzero value $a$ IM and if $f(z)=$ $a \Rightarrow f^{\prime}(z)=a$, then $f \equiv f^{\prime}$. In 2006, Wang [11] replaced the value $a$ by a polynomial $Q \neq \equiv \quad 0$ and obtained the following result: let $f$ be a nonconstant entire function, let $Q$ be a polynomial of degree $q \geq 1$, and let $k>q$ be an integer. If $f$ and $f^{\prime}$ share $Q \mathrm{CM}$ and if $f(z)=Q(z) \Rightarrow f^{(k)}(z)=Q(z)$, then $f \equiv f^{\prime}$.

In 2010, Lü and Yi [12] obtained the following results. 
Theorem 1. Let $f$ be a nonconstant meromorphic function with finitely many poles, and let $R$ be a nonzero rational function. If

$$
\begin{aligned}
f(z) & =R(z) \Longrightarrow f^{\prime}(z)=R(z) \\
f^{\prime}(z) & =R(z) \Longrightarrow|L(f)(z)| \leq(M+o(1))|R(z)|
\end{aligned}
$$

where $M$ is a positive number, then $f$ is of order at most 1 .

Theorem 2. Let $f$ be a nonconstant meromorphic function with finitely many poles, and let $R$ be a nonzero rational function such that $f$ and $R$ have no common poles. If

$$
\begin{aligned}
f(z) & =R(z) \Longrightarrow f^{\prime}(z)=R(z), \\
f^{\prime}(z) & =R(z) \Longrightarrow L(f)(z)=R(z), \\
f^{\prime}(z)-R(z)=0 & \longmapsto f(z)-R(z)=0,
\end{aligned}
$$

then one of the following three cases holds:

(i) $f(z)=b e^{z}$ and $\sum_{n=0}^{k} a_{n}=1$, where $b$ is a nonzero constant;

(ii) $R$ reduces to a constant, say $a$, and $a_{0}+a_{1}+$ $\sum_{n=2}^{k} a_{n} c^{n-1}=1$ for some nonzero constant $c$ such that

$$
f(z)=b e^{c z}-\frac{a(1-c)}{c},
$$

where $b$ is a nonzero constant;

(iii) $R$ is a nonconstant polynomial with $\operatorname{deg} R=l \leq k-2$, $f(z)=\lambda e^{c z}+Q(z)$, and

$$
\begin{aligned}
a_{0}+a_{1} & =1, \\
a_{2} & =a_{3}=\cdots=a_{l}=0, \\
\sum_{n=l+1}^{k} a_{n} c^{n-(l+1)} & =0,
\end{aligned}
$$

where $\lambda, c$ are two nonzero constants and $Q$ is a polynomial such that $c Q-Q^{\prime}=(c-1) R$.

Problem 3. In Theorem 1, we see that $f, f^{\prime}$, and $L(f)$ share one function with zero order. So it is natural to ask what will happen if they share a function of infinite order or positive finite order?

Considering Problem 3, we derive the following results.

Theorem 4 (main theorem). Let $R$ be a nonzero rational function and let $f, \gamma$ be two entire functions. Let $k(\geq 2)$ be an integer and let $L(f)$ be defined as (2). If

$$
\begin{aligned}
f(z) & =\alpha(z) \Longrightarrow f^{\prime}(z)=\alpha(z), \\
f^{\prime}(z) & =\alpha(z) \Longrightarrow|L(f)(z)| \leq(M+o(1))|\alpha(z)|,
\end{aligned}
$$

where $\alpha=\operatorname{Re}^{\gamma}\left(\alpha \neq \equiv \alpha^{\prime}\right)$, and if $\alpha-\alpha^{\prime}$ has at most finitely many zeros, then $\sigma(f) \leq \sigma(\alpha)=\rho(\gamma)$.
The following examples show that our conclusion $\sigma(f) \leq$ $\rho(\gamma)$ really exists and is sharp.

Example 5. Let $f(z)=A e^{z}$, where $A$ is a nonzero constant. Let $\alpha(z)=e^{e^{-z}+z}$. Noting that $f \equiv f^{\prime} \equiv f^{(k)}(k \geq 2)$, we have

$$
\begin{gathered}
f(z)=\alpha(z) \Longrightarrow f^{\prime}(z)=\alpha(z) \\
f^{\prime}(z)=\alpha(z) \Longrightarrow f^{(k)}(z)=\alpha(z) .
\end{gathered}
$$

Obviously, $\alpha(z)-\alpha^{\prime}(z)=e^{e^{-z}}$ has no zeros. Thus it satisfies the assumptions of Theorem 4 and $\sigma(f)=0<\sigma(\alpha)=1$.

Example 6. Let $f(z)=2 e^{z}$ and $\alpha(z)=\left(4 z^{2}-z+2\right) e^{z^{2}}$. Noting that $f \equiv f^{\prime} \equiv f^{(k)}$, then

$$
\begin{gathered}
f(z)=\alpha(z) \Longrightarrow f^{\prime}(z)=\alpha(z), \\
f^{\prime}(z)=\alpha(z) \Longrightarrow f^{(k)}(z)=\alpha(z) .
\end{gathered}
$$

It satisfies the assumptions of Theorem 4 and $\sigma(f)=0$.

Example 7. Let $f(z)=4 z^{2}-8 z+8, \alpha(z)=2 z^{2}$. Noting that $f \not \equiv f^{\prime} \not \equiv f^{\prime \prime}$, then $f(z)-\alpha(z)=2(z-2)^{2}, f^{\prime}(z)-\alpha(z)=$ $-2(z-2)^{2}$, and $f^{\prime \prime}(z)-\alpha(z)=2(2-z)(2+z)$. It is easy to see $f(z)=\alpha(z) \Rightarrow f^{\prime}(z)=\alpha(z)$ and $f^{\prime}(z)=\alpha(z) \Rightarrow$ $f^{\prime \prime}(z)=\alpha(z)$ and $\sigma(f)=\sigma(\alpha)$. It satisfies the assumptions of Theorem 4 and $\sigma(f)=\sigma(\alpha)=0$.

Example 8. Let $f(z)=z^{4} A e^{z}+z^{4}+8 z^{3}+24 z^{2}+48 z+48$ and $\alpha(z)=z^{4}+8 z^{3}+24 z^{2}+48 z+48$, where $A=e^{4}$ is a constant. Differentiating $f$ twice yields $f^{\prime}(z)=\left(z^{4}+4 z^{3}\right) A e^{z}+4 z^{3}+$ $24 z^{2}+48 z+48$ and $f^{\prime \prime}(z)=\left(z^{4}+8 z^{3}+12 z^{2}\right) A e^{z}+12 z^{2}+48 z+$ 48; then $f(z)=\alpha(z) \Rightarrow f^{\prime}(z)=\alpha(z)$ and $f^{\prime}(z)=\alpha(z) \Rightarrow$ $f^{\prime \prime}(z)=\alpha(z)$. Thus $\sigma(f) \leq \sigma(\alpha)$, but $f \not \equiv f^{\prime}$.

Theorem 9 (main theorem). Let $f$ be a meromorphic function with at most finitely many poles, and let $\alpha=\operatorname{Re}^{Q}\left(\alpha \neq \equiv \alpha^{\prime}\right)$, where $R(\neq 0)$ is a rational function and $Q$ is a nonconstant polynomial. Let $k(\geq 2)$ be an integer and let $L(f)$ be defined as (2). Then $\rho(f) \leq \operatorname{deg} Q$, if

$$
\begin{aligned}
f(z)=\alpha(z) & \Longrightarrow f^{\prime}(z)=\alpha(z), \\
f^{\prime}(z)=\alpha(z) & \Longrightarrow|L(f)(z)| \leq(M+o(1))|\alpha(z)| .
\end{aligned}
$$

Example 10. Let $f(z)=4 e^{z}, \alpha(z)=z e^{z+1}$, so $\rho(f)=1$, and $\operatorname{deg} Q=1$. Noting that

$$
\begin{gathered}
f(z)=\alpha(z) \Longrightarrow f^{\prime}(z)=\alpha(z), \\
f^{\prime}(z)=\alpha(z) \Longrightarrow f^{(k)}(z)=\alpha(z),
\end{gathered}
$$

then $\rho(f)=1 \leq \operatorname{deg} Q=1$.

Remark 11. Example 10 illustrates that the conclusion of Theorem 9 really occurs. 
Problem 12. If the rational function $R$ is replaced by a function $\alpha=R e^{Q}$ (here $Q$ is a polynomial) in Theorem 2, what will happen?

Investigating Problem 12, we obtain the following result.

Theorem 13. Let $f$ be a nonconstant transcendental meromorphic function with finitely many poles. Let $\alpha=R^{Q}$ (here $Q$ is a polynomial and $\alpha \neq \alpha^{\prime}$ ) be a function and let $R$ be a nonzero rational function such that $f$ and $R$ have no common poles, and let $L(f)$ be defined as (2). Let $\rho(\alpha)<\rho(f)$. If

$$
\begin{gathered}
f(z)=\alpha(z) \Longrightarrow f^{\prime}(z)=\alpha(z), \\
f^{\prime}(z)=\alpha(z) \Longrightarrow L(f)(z)=\alpha(z),
\end{gathered}
$$

and $f^{\prime}(z)-\alpha(z)=0 \mapsto f(z)-\alpha(z)=0$, then the conclusions of Theorem 2 still hold and $Q(z)$ must be a constant.

\section{Some Lemmas}

In order to prove our theorems, we need the following lemmas.

Normal families, in particular, of holomorphic functions often appear in operator theory on spaces of analytic functions; for example, see in [13, Lemma 3] and in [14, Lemma 4]. Using the same method of the famous Pang-Zalcman Lemma [15, Lemma 2] and the result of Lü et al. [8, Lemma 2.1, page 595], it is easy to obtain the following lemma. It plays an important role in the proof of Theorems 4 and 9.

Lemma 14 (see $[8,15])$. Let $\left\{f_{n}\right\}$ be a family of meromorphic (analytic) functions in the unit disc $\Delta$. If $a_{n} \rightarrow a,|a|<1$, and $f_{n}^{\sharp}\left(a_{n}\right) \rightarrow \infty$, and if there exists $A \geq 1$ such that $\left|f_{n}^{\prime}(z)\right| \leq A$ whenever $f_{n}(z)=0$, then there exist

(i) a subsequence of $f_{n}$ (which we still write as $\left\{f_{n}\right\}$ ),

(ii) points $z_{n} \rightarrow z_{0}\left|z_{0}\right|<1$,

(iii) positive numbers $\rho_{n} \rightarrow 0$,

such that $\rho_{n}^{-1} f_{n}\left(z_{n}+\rho_{n} \xi\right)=g_{n}(\xi) \rightarrow g(\xi)$ locally uniformly, where $g$ is a nonconstant meromorphic (resp., entire) function on $\mathbb{C}$, such that $\rho(g) \leq 2$ (resp., $\rho(g) \leq 1), g^{\sharp}(\xi) \leq g^{\sharp}(0)=$ $A+1$, and

$$
\rho_{n} \leq \frac{M}{f_{n}^{\sharp}\left(a_{n}\right)},
$$

where $M$ is a constant which is independent of $n$.

Here, as usual, $g^{\sharp}(\xi)=\left|g^{\prime}(\xi)\right| /\left(1+|g(\xi)|^{2}\right)$ is the spherical derivative.

The next lemma is an extending result obtained by Lü and Qi in [16].

Lemma 15 (see [16]). Let $f$ be a meromorphic function of hyperorder $\sigma(f)>0$. Then, for any $\epsilon>0$, there exists $a$ sequence $z_{n} \rightarrow \infty$ such that

$$
f^{\sharp}\left(z_{n}\right)>e^{\left|z_{n}\right|^{d-\epsilon}}
$$

for large enough $n$, where $d=\sigma(f)$ if $\sigma(f)<\infty$ or $d$ is an arbitrary positive number.

Lemma 16 (see [5]). Let $f(z)$ be an entire function with $\rho(f)>1$; then for each $0<\mu<\rho(f)-1$, there exist points $a_{n} \rightarrow \infty(n \rightarrow \infty)$, such that

$$
\lim _{n \rightarrow \infty} \frac{f^{\sharp}\left(a_{n}\right)}{\left|a_{n}\right|^{\mu}}=+\infty .
$$

Lemma 17 (see [17]). Let $g$ be a nonconstant entire function with order $\rho(g) \leq 1$, let $k \geq 2$ be an integer, and let $a$ be $a$ nonzero finite value. If $g(z)=0 \Rightarrow g^{\prime}(z)=a$ and $g^{\prime}(z)=$ $a \Rightarrow g^{(k)}(z)=0$, then $g(z)=a\left(z-z_{0}\right)$, where $z_{0}$ is a constant.

Lemma 18 (see [1]). Suppose that $f(z)$ and $g(z)$ are two nonconstant meromorphic functions in the complex plane with $\rho(f)$ and $\rho(g)$ as their orders, respectively. Then

$$
\begin{array}{r}
\rho(f g) \leq \max \{\rho(f), \rho(g)\}, \\
\rho(f+g) \leq \max \{\rho(f), \rho(g)\} .
\end{array}
$$

The following lemma is from the proof of Theorem 2 in [18] (see pages 493-495 in [18]). It plays an important role in the proof of Theorem 13 .

Lemma 19 (see [18]). Let $f$ and $\alpha$ be two meromorphic functions of finite order such that both $f$ and $\alpha$ have finitely many poles, $f$ and $\alpha$ have no common poles, and the order of $\alpha$ is less than the order of $f$. Let $F=f-\alpha$ and $A=\alpha-\alpha^{\prime}$, let $P$ be a nonzero polynomial, and let $Q$ be a polynomial. If $F$ is a solution of differential equation

$$
\frac{F^{\prime}}{F}-\frac{1}{P} e^{Q}=\frac{A}{F},
$$

then $Q$ reduces to a constant and $P$ reduces a constant.

\section{Proof of Theorem 4}

In the proof, we use some ideas of $[8,19-21]$. The proof of Theorem 4 is as follows.

Noting that $\alpha=R e^{\gamma}$, thus $\sigma(\alpha)=\rho(\gamma)$. So we just need to obtain $\sigma(f) \leq \rho(\gamma)$.

On the contrary, suppose that $\sigma(f)>\rho(\gamma)$. Take $d$ such that $\sigma(f)>d>c:=\rho(\gamma)$, and set $H:=f-\alpha$. Then

(I) $H=0 \Rightarrow H^{\prime}=\alpha-\alpha^{\prime}$,

(II) $H^{\prime}=\alpha-\alpha^{\prime} \Rightarrow|L(H)| \leq(M+o(1))|\alpha|+|L(\alpha)|$.

Put $\beta=\alpha-\alpha^{\prime}=\left(R-R^{\prime}-R \gamma^{\prime}\right) e^{\gamma}$ and $\varphi=(M+o(1))|\alpha|+$ $|L(\alpha)|=A\left|P_{R, \gamma} e^{\gamma}\right|$. Here $P_{R, \gamma}=R^{(k)}+R \gamma^{(k)}+R^{(k-1)} \gamma^{\prime}+\cdots+$ $R\left(\gamma^{\prime}\right)^{k}$ is a differential polynomial about $R$ and $\gamma$ and $A$ is a positive number. Set $F=H / \beta$. Obviously, $\sigma(F)=\sigma(f)$. By Lemma 15 , then for $0<\epsilon<(d-c) / 2$, there exists a sequence $w_{n} \rightarrow \infty$ as $n \rightarrow \infty$ such that

$$
F^{\sharp}\left(w_{n}\right)>e^{\left|w_{n}\right|^{\sigma(F)-\epsilon}}>e^{\left|w_{n}\right|^{d-\epsilon}} .
$$


Noting that $\beta=\alpha-\alpha^{\prime}$ has at most finitely many zeros, then there exists a positive number $r$ such that $F$ has no poles in $D=\{z:|z|>r\}$.

In view of $w_{n} \rightarrow \infty$ as $n \rightarrow \infty$, without loss of generality, we may assume $\left|w_{n}\right| \geq r+1$ for all $n$. Define $D_{1}=\{z:|z|<1\}$ and

$$
F_{n}(z)=F\left(w_{n}+z\right)=\frac{H\left(w_{n}+z\right)}{\beta\left(w_{n}+z\right)},
$$

then every $F_{n}$ is analytic in $D_{1}$. Now, fix $z \in D_{1}$. If $F_{n}(z)=0$, then $H\left(w_{n}+z\right)=0$. It is clear from (I) that $H^{\prime}\left(w_{n}+z\right)=$ $\beta\left(w_{n}+z\right)$. Hence (for $n$ large enough)

$$
\begin{aligned}
\left|F_{n}^{\prime}(z)\right| & =\left|\frac{H^{\prime}\left(w_{n}+z\right)}{\beta\left(w_{n}+z\right)}-\frac{H\left(w_{n}+z\right)}{\beta\left(w_{n}+z\right)} \frac{\beta^{\prime}\left(w_{n}+z\right)}{\beta\left(w_{n}+z\right)}\right| \\
& =1 .
\end{aligned}
$$

Also $F_{n}^{\sharp}(0) \rightarrow \infty$ as $n \rightarrow \infty$. It follows from Marty's criterion that $\left(F_{n}\right)_{n}$ is not normal at $z=0$.

Therefore, we can apply Lemma 14. Choosing an appropriate subsequence of $\left(F_{n}\right)_{n}$ if necessary, we may assume that there exist sequences $\left(z_{n}\right)_{n}$ and $\left(\rho_{n}\right)_{n}$ with $\left|z_{n}\right|<r<1$ and $\rho_{n} \rightarrow 0$ such that sequence $\left(g_{n}\right)_{n}$ is defined by

$$
\begin{aligned}
g_{n}(\zeta) & =\rho_{n}^{-1} F_{n}\left(z_{n}+\rho_{n} \zeta\right) \\
& =\rho_{n}^{-1} \frac{H\left(w_{n}+z_{n}+\rho_{n} \zeta\right)}{\beta\left(w_{n}+z_{n}+\rho_{n} \zeta\right)} \longrightarrow g(\zeta)
\end{aligned}
$$

locally uniformly in $\mathbb{C}$, where $g$ is a nonconstant entire function of order at most 1 . Moreover, $g^{\sharp}(\xi) \leq g^{\sharp}(0)=2$ for all $\xi \in \mathbb{C}$ and

$$
\rho_{n} \leq \frac{M}{F_{n}^{\sharp}(0)}=\frac{M}{F^{\sharp}\left(w_{n}\right)} \leq M e^{-\left|w_{n}\right|^{d-\epsilon}}
$$

for a positive number $M$.

We claim that

$$
\rho_{n}^{k-1} \frac{H^{(k)}\left(w_{n}+z_{n}+\rho_{n} \zeta\right)}{\beta\left(w_{n}+z_{n}+\rho_{n} \zeta\right)} \longrightarrow g^{(k)}(\zeta)
$$

locally uniformly in $\mathbb{C}$.

Using the mathematical induction, we prove the claim as follows.

From (21), we have

$$
\begin{aligned}
g_{n}^{\prime}(\zeta)= & \frac{H^{\prime}\left(w_{n}+z_{n}+\rho_{n} \zeta\right)}{\beta\left(w_{n}+z_{n}+\rho_{n} \zeta\right)} \\
& -\frac{H\left(w_{n}+z_{n}+\rho_{n} \zeta\right) \beta^{\prime}\left(w_{n}+z_{n}+\rho_{n} \zeta\right)}{\beta\left(w_{n}+z_{n}+\rho_{n} \zeta\right) \beta\left(w_{n}+z_{n}+\rho_{n} \zeta\right)} \\
= & \frac{H^{\prime}\left(w_{n}+z_{n}+\rho_{n} \zeta\right)}{\beta\left(w_{n}+z_{n}+\rho_{n} \zeta\right)} \\
& -\rho_{n} g_{n}(\zeta) \frac{\beta^{\prime}\left(w_{n}+z_{n}+\rho_{n} \zeta\right)}{\beta\left(w_{n}+z_{n}+\rho_{n} \zeta\right)} \longrightarrow g^{\prime}(\zeta)
\end{aligned}
$$

Noting that $\beta=\alpha-\alpha^{\prime}=\left(R-R^{\prime}-R \gamma^{\prime}\right) e^{\gamma}$ and $\varphi=\alpha-\alpha^{(k)}=$ $P_{R, \gamma} e^{\gamma}$, we have $\beta^{\prime} / \beta=\left(R^{\prime}+R \gamma^{\prime}-R^{\prime \prime}-2 R^{\prime} \gamma^{\prime}-R \gamma^{\prime \prime}-R \gamma^{\prime 2}\right) /(R-$ $\left.R^{\prime}-R \gamma^{\prime}\right)$ and $\rho\left(\gamma^{\prime \prime}\right)=\rho\left(\gamma^{\prime}\right)=\rho\left(\gamma^{\prime 2}\right)=\rho(\gamma)=c$. In view of the definition of order, we have

$$
\begin{aligned}
& \left|\frac{\beta^{\prime}}{\beta}\right|_{z=w_{n}+z_{n}+\rho_{n} \zeta} \mid \\
& =\left|\frac{R^{\prime}+R \gamma^{\prime}-R^{\prime \prime}-2 R^{\prime} \gamma^{\prime}-R \gamma^{\prime \prime}-R \gamma^{\prime 2}}{R-R^{\prime}-R \gamma^{\prime}}\right|_{z=w_{n}+z_{n}+\rho_{n} \zeta} \mid \\
& \leq\left|w_{n}\right|^{q} M\left(\left|w_{n}+z_{n}+\rho_{n} \zeta\right|, \gamma^{\prime}\right) \\
& \leq\left|w_{n}\right|^{q} M\left(2\left|w_{n}\right|, \gamma^{\prime}\right) \leq\left|w_{n}\right|^{q} e^{A\left|w_{n}\right|^{c+\varepsilon}},
\end{aligned}
$$

where $A$ is a positive constant and $q$ is an integer. Noting that $0<\epsilon<(d-c) / 2$, we have $d-\epsilon>c+\epsilon$. Then, combining (22) and (25) yields

$$
\begin{aligned}
& \left|\frac{H\left(w_{n}+z_{n}+\rho_{n} \zeta\right) \beta^{\prime}\left(w_{n}+z_{n}+\rho_{n} \zeta\right)}{\beta\left(w_{n}+z_{n}+\rho_{n} \zeta\right) \beta\left(w_{n}+z_{n}+\rho_{n} \zeta\right)}\right| \\
& =\left|\rho_{n} g_{n}(\zeta) \frac{\beta^{\prime}\left(w_{n}+z_{n}+\rho_{n} \zeta\right)}{\beta\left(w_{n}+z_{n}+\rho_{n} \zeta\right)}\right| \\
& \leq M\left|g_{n}(\zeta)\right|\left|w_{n}\right|^{q} e^{A\left|w_{n}\right|^{c+\varepsilon}-\left|w_{n}\right|^{d-\epsilon}} \longrightarrow 0, \\
& \text { as } n \longrightarrow \infty .
\end{aligned}
$$

From (24) and (26), we deduce that

$$
\frac{H^{\prime}\left(w_{n}+z_{n}+\rho_{n} \zeta\right)}{\beta\left(w_{n}+z_{n}+\rho_{n} \zeta\right)} \longrightarrow g^{\prime}(\zeta)
$$

locally uniformly in $\mathbb{C}$, which implies that the claim is right when $k=1$.

We assume that the claim is also right when $k=l$; that is,

$$
\rho_{n}^{l-1} \frac{H^{(l)}\left(w_{n}+z_{n}+\rho_{n} \zeta\right)}{\beta\left(w_{n}+z_{n}+\rho_{n} \zeta\right)} \longrightarrow g^{(l)}(\zeta)
$$

locally uniformly in $\mathbb{C}$. Then

$$
\text { Define } G_{n}(\zeta)=\rho_{n}^{l-1}\left(H^{(l)}\left(w_{n}+z_{n}+\rho_{n} \zeta\right) / \beta\left(w_{n}+z_{n}+\rho_{n} \zeta\right)\right) \text {. }
$$

$G_{n}^{\prime}(\zeta)$

$$
\begin{aligned}
= & \rho_{n}^{l} \frac{H^{(l+1)}\left(w_{n}+z_{n}+\rho_{n} \zeta\right)}{\beta\left(w_{n}+z_{n}+\rho_{n} \zeta\right)} \\
& -\rho_{n}^{l} \frac{H^{(l)}\left(w_{n}+z_{n}+\rho_{n} \zeta\right) \beta^{\prime}\left(w_{n}+z_{n}+\rho_{n} \zeta\right)}{\beta\left(w_{n}+z_{n}+\rho_{n} \zeta\right) \beta\left(w_{n}+z_{n}+\rho_{n} \zeta\right)} \\
= & \rho_{n}^{l} \frac{H^{(l+1)}\left(w_{n}+z_{n}+\rho_{n} \zeta\right)}{\beta\left(w_{n}+z_{n}+\rho_{n} \zeta\right)} \\
& -\rho_{n} G_{n}(\zeta) \frac{\beta^{\prime}\left(w_{n}+z_{n}+\rho_{n} \zeta\right)}{\beta\left(w_{n}+z_{n}+\rho_{n} \zeta\right)} \longrightarrow g^{(l+1)}(\zeta)
\end{aligned}
$$


locally uniformly in $\mathbb{C}$. Note the fact that

$$
\rho_{n} G_{n}(\zeta) \frac{\beta^{\prime}\left(w_{n}+z_{n}+\rho_{n} \zeta\right)}{\beta\left(w_{n}+z_{n}+\rho_{n} \zeta\right)} \longrightarrow 0 .
$$

We immediately derive that

$$
\rho_{n}^{l} \frac{H^{(l+1)}\left(w_{n}+z_{n}+\rho_{n} \zeta\right)}{\beta\left(w_{n}+z_{n}+\rho_{n} \zeta\right)} \longrightarrow g^{(l+1)}(\zeta)
$$

locally uniformly in $\mathbb{C}$, which finishes the proof of the claim.

Furthermore, we have

$$
\begin{aligned}
& \frac{\rho_{n}^{k-1} L(H)\left(w_{n}+z_{n}+\rho_{n} \zeta\right)}{a_{k} \beta\left(w_{n}+z_{n}+\rho_{n} \zeta\right)} \\
& \quad=\frac{\rho_{n}^{k-1} H^{(k)}\left(w_{n}+z_{n}+\rho_{n} \zeta\right)}{\beta\left(w_{n}+z_{n}+\rho_{n} \zeta\right)}+\cdots \\
& \quad+\frac{\rho_{n}^{k-1} a_{0} H\left(w_{n}+z_{n}+\rho_{n} \zeta\right)}{a_{k} \beta\left(w_{n}+z_{n}+\rho_{n} \zeta\right)} \longrightarrow g^{(k)}(\zeta)
\end{aligned}
$$

locally uniformly in $\mathbb{C}$.

We claim that

(1) $g(\zeta)=0 \Rightarrow g^{\prime}(\zeta)=1$,

(2) $g^{\prime}(\zeta)=1 \Rightarrow g^{(k)}(\zeta)=0$.

Suppose that $g\left(\zeta_{0}\right)=0$; then by Hurwitz's theorem there exist $\zeta_{n}, \zeta_{n} \rightarrow \zeta_{0}$, such that (for $n$ sufficiently large)

$$
g_{n}\left(\zeta_{n}\right)=\rho_{n}^{-1} \frac{H\left(w_{n}+z_{n}+\rho_{n} \zeta_{n}\right)}{\beta\left(w_{n}+z_{n}+\rho_{n} \zeta_{n}\right)}=0 .
$$

Thus $H\left(w_{n}+z_{n}+\rho_{n} \zeta_{n}\right)=0$; by (I) we have

$$
H^{\prime}\left(w_{n}+z_{n}+\rho_{n} \zeta_{n}\right)=\beta\left(w_{n}+z_{n}+\rho_{n} \zeta_{n}\right) \text {. }
$$

By (27), we derive that

$$
g^{\prime}\left(\zeta_{0}\right)=\lim _{n \rightarrow \infty} \frac{H^{\prime}\left(w_{n}+z_{n}+\rho_{n} \zeta_{n}\right)}{\beta\left(w_{n}+z_{n}+\rho_{n} \zeta_{n}\right)}=1,
$$

which implies that $g(\zeta)=0 \Rightarrow g^{\prime}(\zeta)=1$.

To prove (2), suppose that $g^{\prime}\left(\eta_{0}\right)=1$. We know $g^{\prime} \not \equiv 1$; otherwise $g^{\sharp}(0) \leq 1<2$ is a contradiction. Hence by (27) and Hurwitz's theorem, there exist $\eta_{n} \rightarrow \eta_{0}$ such that (for $n$ sufficiently large)

$$
H^{\prime}\left(w_{n}+z_{n}+\rho_{n} \eta_{n}\right)=\beta\left(w_{n}+z_{n}+\rho_{n} \eta_{n}\right) .
$$

It is obvious from (II) that $\left|L(H)\left(w_{n}+z_{n}+\rho_{n} \eta_{n}\right)\right| \leq$ $A\left|P_{R, \gamma} e^{\gamma}\left(w_{n}+z_{n}+\rho_{n} \eta_{n}\right)\right|$. By (23), (32), and $\rho\left(\gamma^{(k)}\right)=\rho\left(\gamma^{\prime}\right)=$ $\rho\left(\left(\gamma^{\prime}\right)^{k}\right)=\rho(\gamma)=c$, similarly with (25) and (26), we obtain

$$
\begin{aligned}
& g^{(k)}\left(\eta_{0}\right)=\lim _{n \rightarrow \infty} \frac{\rho_{n}^{k-1} L(H)\left(w_{n}+z_{n}+\rho_{n} \eta_{n}\right)}{a_{k} \beta\left(w_{n}+z_{n}+\rho_{n} \eta_{n}\right)}=0 \\
& \quad \leq \lim _{n \rightarrow \infty} \rho_{n}^{k-1} A\left|\frac{P_{R, \gamma}}{a_{k}\left(R-R^{\prime}-R \gamma^{\prime}\right)}\right|_{z=w_{n}+z_{n}+\rho_{n} \eta_{n}} \mid=0
\end{aligned}
$$

which yields (2). By Lemma 17, it is easy to deduce that $g(\zeta)=$ $\zeta-b_{0}$, where $b_{0}$ is a constant; then $g^{\sharp}(0) \leq 1<2$, which is also a contradiction.

Thus, we complete the proof of Theorem 4 .

\section{Proof of Theorem 9}

Set $H=f-\alpha$. Then

(I) $H(z)=0 \Rightarrow H^{\prime}(z)=\alpha(z)-\alpha^{\prime}(z)$,

(II) $H^{\prime}(z)=\alpha-\alpha^{\prime} \Rightarrow|L(H)| \leq(M+o(1))|\alpha|+|L(\alpha)|$.

Put $\beta=\alpha-\alpha^{\prime}=\left(R-R^{\prime}-R Q^{\prime}\right) e^{Q}=R_{1} e^{Q}$ and $\varphi=$ $(M+o(1))|\alpha|+|L(\alpha)|=A\left|R_{2} e^{Q}\right|$. Here $R_{2}=R^{(k)}+R Q^{(k)}+$ $R^{(k-1)} Q^{\prime}+\cdots+R\left(Q^{\prime}\right)^{k}, R_{1}(\not \equiv 0)$ and $R_{2}$ are two rational functions, $\operatorname{deg} R_{2}=\operatorname{deg} R Q^{\prime k}, A$ is a positive number. Set $F=$ $H / \beta$.

Noting that $\beta=\alpha-\alpha^{\prime}$ has at most finitely many zeros and $f$ has finitely many poles, then there exists a positive number $r$ such that $F$ has no poles in $D=\{z:|z|>r\}$.

If $\rho(F)>\operatorname{deg} Q$, by Lemma 16 , for every $0<\mu<\rho(F)-1$, there exist $w_{n} \rightarrow \infty$, such that, for $n \rightarrow \infty$,

$$
\lim _{n \rightarrow \infty} \frac{F^{\sharp}\left(w_{n}\right)}{\left|w_{n}\right|^{\mu}}=+\infty .
$$

In view of $w_{n} \rightarrow \infty$ as $n \rightarrow \infty$, without loss of generality, we may assume $\left|w_{n}\right| \geq r+1$ for all $n$. Define $D_{1}=\{z:|z|<1\}$ and

$$
F_{n}(z)=F\left(w_{n}+z\right)=\frac{H\left(w_{n}+z\right)}{\beta\left(w_{n}+z\right)} ;
$$

then every $F_{n}$ is analytic in $D_{1}$. Now, fix $z \in D_{1}$. If $F_{n}(z)=0$, then $H\left(w_{n}+z\right)=0$. It is clear from (I) that $H^{\prime}\left(w_{n}+z\right)=$ $\beta\left(w_{n}+z\right)$. Hence (for $n$ large enough)

$$
\begin{aligned}
\left|F_{n}^{\prime}(z)\right| & =\left|\frac{H^{\prime}\left(w_{n}+z\right)}{\beta\left(w_{n}+z\right)}-\frac{H\left(w_{n}+z\right)}{\beta\left(w_{n}+z\right)} \frac{\beta^{\prime}\left(w_{n}+z\right)}{\beta\left(w_{n}+z\right)}\right| \\
& =1
\end{aligned}
$$

Also $F_{n}^{\sharp}(0) \rightarrow \infty$ as $n \rightarrow \infty$. It follows from Marty's criterion that $\left(F_{n}\right)_{n}$ is not normal at $z=0$.

Therefore, we can apply Lemma 14. Choosing an appropriate subsequence of $\left(F_{n}\right)_{n}$ if necessary, we may assume that there exist sequences $\left(z_{n}\right)_{n}$ and $\left(\rho_{n}\right)_{n}$ with $\left|z_{n}\right|<r<1$ and $\rho_{n} \rightarrow 0$ such that sequences $\left(g_{n}\right)_{n}$ is defined by

$$
g_{n}(\zeta)=\rho_{n}^{-1} F_{n}\left(z_{n}+\rho_{n} \zeta\right) \longrightarrow g(\zeta)
$$

locally uniformly in $\mathbb{C}$, where $g$ is a nonconstant entire function of order at most 1 . Moreover, $g^{\sharp}(\xi) \leq g^{\sharp}(0)=2$ for all $\xi \in \mathbb{C}$ and

$$
\rho_{n} \leq \frac{M}{F_{n}^{\sharp}(0)}=\frac{M}{F^{\sharp}\left(w_{n}\right)} \leq M\left|w_{n}\right|^{-\mu-\epsilon}
$$

for a positive number $M$.

We claim that

$$
\rho_{n}^{k-1} \frac{H^{(k)}\left(w_{n}+z_{n}+\rho_{n} \zeta\right)}{\beta\left(w_{n}+z_{n}+\rho_{n} \zeta\right)} \longrightarrow g^{(k)}(\zeta)
$$

locally uniformly in $\mathbb{C}$. 
Using the mathematical induction, we prove the claim as follows.

From (41), we have

$$
\begin{aligned}
g_{n}^{\prime}(\zeta)= & \frac{H^{\prime}\left(w_{n}+z_{n}+\rho_{n} \zeta\right)}{\beta\left(w_{n}+z_{n}+\rho_{n} \zeta\right)} \\
& -\frac{H\left(w_{n}+z_{n}+\rho_{n} \zeta\right) \beta^{\prime}\left(w_{n}+z_{n}+\rho_{n} \zeta\right)}{\beta\left(w_{n}+z_{n}+\rho_{n} \zeta\right) \beta\left(w_{n}+z_{n}+\rho_{n} \zeta\right)} \\
= & \frac{H^{\prime}\left(w_{n}+z_{n}+\rho_{n} \zeta\right)}{\beta\left(w_{n}+z_{n}+\rho_{n} \zeta\right)} \\
& -\rho_{n} g_{n}(\zeta) \frac{\beta^{\prime}\left(w_{n}+z_{n}+\rho_{n} \zeta\right)}{\beta\left(w_{n}+z_{n}+\rho_{n} \zeta\right)} \longrightarrow g^{\prime}(\zeta)
\end{aligned}
$$

Noting that $\beta=\alpha-\alpha^{\prime}=R_{1} e^{Q}$ and $\varphi=(M+o(1))|\alpha|+$ $|L(\alpha)|=A\left|R_{2} e^{Q}\right|$, we have

$$
\begin{aligned}
& \left.\left|\frac{\beta^{\prime}}{\beta}\right|_{z=w_{n}+z_{n}+\rho_{n} \zeta}|=| \frac{R_{1}^{\prime}+R_{1} Q^{\prime}}{R_{1}}\right|_{z=w_{n}+z_{n}+\rho_{n} \zeta} \mid \\
& =\left|\frac{R^{\prime}-R^{\prime \prime}-2 R^{\prime} Q^{\prime}-R Q^{\prime \prime}+R Q^{\prime}-R Q^{\prime 2}}{R-R^{\prime}-R Q^{\prime}}\right|_{z=w_{n}+z_{n}+\rho_{n} \zeta} \mid \\
& =O\left(\left|w_{n}\right|^{l_{1}}\right) .
\end{aligned}
$$

By (38) and (42), combining with $\rho(F)>\operatorname{deg} Q$ and $0<$ $\mu<\rho(F)-1$, we deduce that

$$
\lim _{n \rightarrow \infty} w_{n}^{l_{1}} \rho_{n} \leq \lim _{n \rightarrow \infty} M\left|w_{n}\right|^{l_{1}-(\mu+\epsilon)}=0,
$$

where $l_{1}=\operatorname{deg}\left(\left(R^{\prime}-R^{\prime \prime}-2 R^{\prime} Q^{\prime}-R Q^{\prime \prime}+R Q^{\prime}-R Q^{\prime 2}\right) /\left(R-R^{\prime}-\right.\right.$ $\left.\left.R Q^{\prime}\right)\right)=\operatorname{deg}\left(\left(R^{\prime} / R-R^{\prime \prime} / R-2\left(R^{\prime} / R\right) Q^{\prime}-Q^{\prime \prime}+Q^{\prime}-Q^{\prime 2}\right) /(1-\right.$ $\left.R^{\prime}\left(R-Q^{\prime}\right)\right)=\operatorname{deg} Q^{\prime}$ is a fixed constant. Then, combining (38), (42), (45), and (46) yields

$$
\begin{aligned}
& \left|\frac{H\left(w_{n}+z_{n}+\rho_{n} \zeta\right) \beta^{\prime}\left(w_{n}+z_{n}+\rho_{n} \zeta\right)}{\beta\left(w_{n}+z_{n}+\rho_{n} \zeta\right) \beta\left(w_{n}+z_{n}+\rho_{n} \zeta\right)}\right| \\
& \quad=\left|\rho_{n} g_{n}(\zeta) \frac{\beta^{\prime}\left(w_{n}+z_{n}+\rho_{n} \zeta\right)}{\beta\left(w_{n}+z_{n}+\rho_{n} \zeta\right)}\right| \\
& \quad \leq M\left|g_{n}(\zeta)\right|\left|w_{n}\right|^{l_{1}-\mu-\epsilon} \longrightarrow 0, \text { as } n \longrightarrow \infty .
\end{aligned}
$$

From (44) and (47), we deduce that

$$
\frac{H^{\prime}\left(w_{n}+z_{n}+\rho_{n} \zeta\right)}{\beta\left(w_{n}+z_{n}+\rho_{n} \zeta\right)} \longrightarrow g^{\prime}(\zeta)
$$

locally uniformly in $\mathbb{C}$, which implies that the claim is right when $k=1$.

We assume that the claim is also right when $k=l$; that is,

$$
\rho_{n}^{l-1} \frac{H^{(l)}\left(w_{n}+z_{n}+\rho_{n} \zeta\right)}{\beta\left(w_{n}+z_{n}+\rho_{n} \zeta\right)} \longrightarrow g^{(l)}(\zeta)
$$

locally uniformly in $\mathbb{C}$.
Define $G_{n}(\zeta)=\rho_{n}^{l-1}\left(H^{(l)}\left(w_{n}+z_{n}+\rho_{n} \zeta\right) / \beta\left(w_{n}+z_{n}+\rho_{n} \zeta\right)\right)$ Then

$$
\begin{aligned}
G_{n}^{\prime}(\zeta) & \\
= & \rho_{n}^{l} \frac{H^{(l+1)}\left(w_{n}+z_{n}+\rho_{n} \zeta\right)}{\beta\left(w_{n}+z_{n}+\rho_{n} \zeta\right)} \\
& -\rho_{n}^{l} \frac{H^{(l)}\left(w_{n}+z_{n}+\rho_{n} \zeta\right) \beta^{\prime}\left(w_{n}+z_{n}+\rho_{n} \zeta\right)}{\beta\left(w_{n}+z_{n}+\rho_{n} \zeta\right) \beta\left(w_{n}+z_{n}+\rho_{n} \zeta\right)} \\
= & \rho_{n}^{l} \frac{H^{(l+1)}\left(w_{n}+z_{n}+\rho_{n} \zeta\right)}{\beta\left(w_{n}+z_{n}+\rho_{n} \zeta\right)} \\
& -\rho_{n} G_{n}(\zeta) \frac{\beta^{\prime}\left(w_{n}+z_{n}+\rho_{n} \zeta\right)}{\beta\left(w_{n}+z_{n}+\rho_{n} \zeta\right)} \longrightarrow g^{(l+1)}(\zeta)
\end{aligned}
$$

locally uniformly in $\mathbb{C}$. Note the fact that

$$
\rho_{n} G_{n}(\zeta) \frac{\beta^{\prime}\left(w_{n}+z_{n}+\rho_{n} \zeta\right)}{\beta\left(w_{n}+z_{n}+\rho_{n} \zeta\right)} \longrightarrow 0 .
$$

We immediately derive that

$$
\rho_{n}^{l} \frac{H^{(l+1)}\left(w_{n}+z_{n}+\rho_{n} \zeta\right)}{\beta\left(w_{n}+z_{n}+\rho_{n} \zeta\right)} \longrightarrow g^{(l+1)}(\zeta)
$$

locally uniformly in $\mathbb{C}$, which finishes the proof of the claim. Furthermore, we have

$$
\begin{aligned}
& \frac{\rho_{n}^{k-1} L(H)\left(w_{n}+z_{n}+\rho_{n} \zeta\right)}{a_{k} \beta\left(w_{n}+z_{n}+\rho_{n} \zeta\right)} \\
& =\frac{\rho_{n}^{k-1} H^{(k)}\left(w_{n}+z_{n}+\rho_{n} \zeta\right)}{\beta\left(w_{n}+z_{n}+\rho_{n} \zeta\right)}+\cdots \\
& \quad+\frac{\rho_{n}^{k-1} a_{1} H^{\prime}\left(w_{n}+z_{n}+\rho_{n} \zeta\right)}{a_{k} \beta\left(w_{n}+z_{n}+\rho_{n} \zeta\right)} \\
& +\frac{\rho_{n}^{k-1} a_{0} H\left(w_{n}+z_{n}+\rho_{n} \zeta\right)}{a_{k} \beta\left(w_{n}+z_{n}+\rho_{n} \zeta\right)} \longrightarrow g^{(k)}(\zeta)
\end{aligned}
$$

locally uniformly in $\mathbb{C}$.

We claim that

(1) $g(\zeta)=0 \Rightarrow g^{\prime}(\zeta)=1$,

(2) $g^{\prime}(\zeta)=1 \Rightarrow g^{(k)}(\zeta)=0$.

The proof of (1) is exactly the same as in the proof of Theorem 4. To prove (2), just replace (53) in the previous proof by

$$
\begin{aligned}
g^{(k)} & \left(\eta_{0}\right)=\lim _{n \rightarrow \infty} \frac{\rho_{n}^{k-1} L(H)\left(w_{n}+z_{n}+\rho_{n} \eta_{n}\right)}{a_{k} \beta\left(w_{n}+z_{n}+\rho_{n} \eta_{n}\right)} \\
& \leq \lim _{n \rightarrow \infty} \rho_{n}^{k-1}\left|\frac{\varphi\left(w_{n}+z_{n}+\rho_{n} \eta_{n}\right)}{a_{k} \beta\left(w_{n}+z_{n}+\rho_{n} \eta_{n}\right)}\right| \\
& =\lim _{n \rightarrow \infty} \rho_{n}^{k-1}\left|\frac{R_{2}\left(w_{n}+z_{n}+\rho_{n} \eta_{n}\right)}{a_{k}\left(R-R^{\prime}-R Q^{\prime}\right)\left(w_{n}+z_{n}+\rho_{n} \eta_{n}\right)}\right| \\
& =\lim _{n \rightarrow \infty} \rho_{n}^{k-1}\left(O\left(\left|w_{n}\right|^{l_{2}}\right)\right) .
\end{aligned}
$$


Here $l_{2}=\operatorname{deg}\left(R_{2} /\left(R-R^{\prime}-R Q^{\prime}\right)\right)=\operatorname{deg}\left(R_{2} / R /\left(1-R^{\prime} / R-\right.\right.$ $\left.\left.Q^{\prime}\right)\right)=\operatorname{deg}\left(Q^{\prime}\right)^{k-1}$ is also a fixed constant.

By (38) and (42), combining with $\rho(F)>\operatorname{deg} Q$ and $0<$ $\mu<\rho(F)-1$, we deduce that

$$
\lim _{n \rightarrow \infty} w_{n}^{l_{2}} \rho_{n}^{k-1} \leq \lim _{n \rightarrow \infty} M\left|w_{n}\right|^{l_{2}-(k-1)(\mu+\epsilon)}=0,
$$

which yields (2). From Lemma 17, it is easy to deduce that $g(\zeta)=\zeta-b_{0}$, where $b_{0}$ is a constant; then $g^{\sharp}(0) \leq 1<2$, which is also a contradiction. $\operatorname{deg} Q$.

So $\rho(F) \leq \operatorname{deg} Q$. Next we will prove $\rho(f) \leq \rho(F) \leq$

If $\rho(\alpha)<\rho(f)$, noting that $\rho\left(\alpha-\alpha^{\prime}\right) \leq \rho(\alpha)$, by Lemma 18, then $\rho\left(F\left(\alpha-\alpha^{\prime}\right)\right) \leq \max \{\rho(F), \rho(\alpha)\}$. Due to $F=(f-\alpha) /(\alpha-$ $\left.\alpha^{\prime}\right)$, we have $f=\alpha+F\left(\alpha-\alpha^{\prime}\right)$. Thus, by Lemma 18, $\rho(f) \leq$ $\max \left\{\rho(\alpha), \rho\left(F\left(\alpha-\alpha^{\prime}\right)\right)\right\} \leq \max \{\rho(\alpha), \rho(F)\}$. Then, it follows from $\rho(\alpha)<\rho(f)$ that $\rho(f) \leq \rho(F) \leq \operatorname{deg} Q$.

If $\rho(\alpha)=\rho(f)$, noting that $\rho(\alpha)=\operatorname{deg} Q$, we obtain $\rho(f)=\rho(\alpha)=\operatorname{deg} Q$.

If $\rho(\alpha)>\rho(f)$, due to $F=(f-\alpha) /\left(\alpha-\alpha^{\prime}\right)=\left(f-R e^{Q}\right) /$ $R_{1} e^{Q}=f / R_{1} e^{Q}-R / R_{1}$, hence $f=\left(F+R / R_{1}\right) R_{1} e^{Q}$. Because $R / R_{1}$ is a rational function, we get $\rho\left(R / R_{1}\right)=\rho\left(R_{1}\right)=0$. By Lemma 18, we may obtain $\operatorname{deg} Q=\rho\left(R e^{Q}\right)=\rho(\alpha)<\rho(f) \leq$ $\max \left\{\rho\left(F+R / R_{1}\right), \rho\left(R_{1} e^{Q}\right)\right\} \leq \max \left\{\rho(F), \rho\left(R_{1} e^{Q}\right)\right\} \leq \operatorname{deg} Q$, a contradiction.

At last, we obtain $\rho(f) \leq \operatorname{deg} Q$.

Thus, we complete the proof of Theorem 9.

\section{Proof of Theorem 13}

Now we distinguish two cases.

Case 1 (if $Q$ is a nonconstant polynomial). By the assumption of Theorem 13 and the result of Theorem 9, we easily deduce that $f$ is of order at most $\operatorname{deg} Q$. Define

$$
\mu=\frac{f^{\prime}-\alpha}{f-\alpha} .
$$

The fact that $f$ is transcendental and $\rho(\alpha)<\rho(f)$ implies $\mu \neq \equiv 0$.

Because of $f^{\prime}(z)-\alpha(z)=0 \mapsto f(z)-\alpha(z)=0$, it is easy to obtain that $\mu$ has no zeros. With the assumption $f(z)=\alpha(z) \Rightarrow f^{\prime}(z)=\alpha(z)$, we derive that $f-\alpha$ has finitely many multiple zeros. We know that the possible poles of $\mu$ are from the multiple zeros of $f-\alpha$ and the poles of $f$; thus $\mu$ has finitely many poles. Moreover, from (56), we have $\rho(\mu) \leq \rho(f) \leq \operatorname{deg} Q$. Therefore, we can set

$$
\mu=\frac{1}{P(z)} e^{Q^{*}(z)}
$$

where $P$ is a nonzero polynomial and $\operatorname{deg} Q^{*}$ is at most $\operatorname{deg} Q$.

Set $F=f-\alpha$ and $A=\alpha-\alpha^{\prime}$. We obtain

$$
\frac{F^{\prime}}{F}-\frac{1}{P} e^{Q^{*}}=\frac{A}{F} .
$$

By Lemma 19, we get $Q^{*}$ and $P$; both are constants. Thus $\mu$ is a nonzero constant. Set $\mu=c$, rewriting (56) as

$$
f^{\prime}=c f+(1-c) \alpha=c f+H
$$

where $H=(1-c) \alpha$.

If $c=1$, then $f=f^{\prime}$ and $f(z)=b e^{z}$, where $b$ is a nonzero constant. So $\rho(f)=1$. Since $Q$ is a nonconstant polynomial, we get $\rho(\alpha)=\rho\left(R e^{\mathrm{Q}}\right) \geq 1$. It is a contradiction with the condition $\rho(\alpha)<\rho(f)$.

Next, we consider $c \neq 1$. Differentiating (59) $k-1$ times yields

$$
\begin{aligned}
f^{(k)}= & c^{k} f+c^{k-1} H+c^{k-2} H^{\prime}+\cdots+c H^{(k-2)} \\
& +H^{(k-1)} .
\end{aligned}
$$

Furthermore, we get

$$
\begin{aligned}
L(f)= & \sum_{n=0}^{k} a_{n} c^{n} f+\sum_{n=1}^{k} a_{n} c^{n-1} H+\sum_{n=2}^{k} a_{n} c^{n-2} H^{\prime}+\cdots \\
& +\sum_{n=p+1}^{k} a_{n} c^{n-(p+1)} H^{(p)}+\cdots+a_{k} H^{(k-1)} .
\end{aligned}
$$

Set

$$
\begin{aligned}
\phi= & \sum_{n=0}^{k} a_{n} c^{n} \alpha+\sum_{n=1}^{k} a_{n} c^{n-1}(1-c) \alpha \\
& +\sum_{n=2}^{k} a_{n} c^{n-2}(1-c) \alpha^{\prime}+\cdots \\
& +\sum_{n=p+1}^{k} a_{n} c^{n-(p+1)}(1-c) \alpha^{(p)}+\cdots \\
& +a_{k}(1-c) \alpha^{(k-1)} \\
= & \left(a_{0}+a_{1}+\sum_{n=2}^{k} a_{n} c^{n-1}\right) \alpha+\sum_{n=2}^{k} a_{n} c^{n-2}(1-c) \alpha^{\prime} \\
& +\cdots+\sum_{n=p+1}^{k} a_{n} c^{n-(p+1)}(1-c) \alpha^{(p)}+\cdots \\
& +a_{k}(1-c) \alpha^{(k-1)}=R_{1} e^{\mathrm{Q}} .
\end{aligned}
$$

Obviously, $R_{1}$ is a rational function.

Suppose $z_{0}$ is a zero of $f-\alpha$. Substituting $H=(1-c) \alpha$ and $z_{0}$ into (61), we get $\alpha\left(z_{0}\right)=\phi\left(z_{0}\right)$; this shows that $z_{0}$ is also a zero of $\alpha-\phi$. 
Next, we will prove that $\alpha \equiv \phi$. Otherwise, we assume that $\alpha \neq \equiv \phi$. Combining the above discussion and the fact that $f-\alpha$ has finitely many multiple zeros yields

$$
\begin{aligned}
N\left(r, \frac{1}{f-\alpha}\right) & =N_{1)}\left(r, \frac{1}{f-\alpha}\right)+N_{(2}\left(r, \frac{1}{f-\alpha}\right) \\
& \leq N\left(r, \frac{1}{\alpha-\phi}\right)+O(\log r) \\
& =N\left(r, \frac{1}{\left(R-R_{1}\right) e^{\mathrm{Q}}}\right)+O(\log r) \\
& =O(\log r),
\end{aligned}
$$

and it implies that $f-\alpha$ has finitely many zeros. Thus we can set $f-\alpha=R_{2}(z) e^{Q_{1}}$, where $R_{2}$ is a rational function and $Q_{1}$ is a polynomial and degree at most $\operatorname{deg} Q$. Differentiating the above equation leads to

$$
f^{\prime}(z)=\alpha^{\prime}(z)+\left(R_{2}^{\prime}+Q_{1}^{\prime} R_{2}\right) e^{Q_{1}} .
$$

By the fact that $f-\alpha$ has finitely many zeros and the assumption $f^{\prime}(z)=\alpha(z) \mapsto f(z)=\alpha(z)$, we deduce that $f^{\prime}-\alpha$ also has finitely many zeros. Noting that

$$
\begin{aligned}
f^{\prime}(z)-\alpha(z)= & \alpha^{\prime}(z)-\alpha(z) \\
& +\left(R_{2}^{\prime}(z)+Q_{1}^{\prime}(z) R_{2}(z)\right) e^{Q_{1}(z)} ;
\end{aligned}
$$

thus $\alpha-\alpha^{\prime}$ or $R_{2}^{\prime}+R_{2} Q_{1}^{\prime} \equiv 0$. By the assumptions of Theorem $13, \alpha \neq \alpha^{\prime}$, this is impossible.

If $R_{2}^{\prime}+Q_{1}^{\prime} R_{2}=0$, then $R_{2}^{\prime} / R_{2}=-Q_{1}^{\prime}$. By the definition, we get $\operatorname{deg}\left(R_{2}^{\prime} / R_{2}\right)=0$ and also $\operatorname{deg} Q_{1}^{\prime}=0$. So we may set $Q_{1}=b z+d$ or $Q_{1}=a$, where $b$ is a nonzero constant and $a, d$ are two constants.

If $Q_{1}=a$, then $f=\alpha+a$. So $\rho(f)=\rho(\alpha)$. It is a contradiction about the condition $\rho(\alpha)<\rho(f)$.

If $Q_{1}=b z+d$, then $f-\alpha=R_{2}(z) e^{b z+d}$. So (64) changes to

$$
f^{\prime}=\alpha^{\prime}+\left(R_{2}^{\prime}+b R_{2}\right) e^{b z+d},
$$

and (65) turns into

$$
f^{\prime}-\alpha=\alpha^{\prime}-\alpha+\left(R_{2}^{\prime}+b R_{2}\right) e^{b z+d} .
$$

Thus $\alpha-\alpha^{\prime}=0$ or $R_{2}^{\prime}+R_{2} b=0$. Also $\alpha \neq \alpha^{\prime}$ is impossible. If $R_{2}^{\prime}+R_{2} b=0$, then $b=0$, a contradiction.

At last, we complete the proof of

$$
\alpha \equiv \phi
$$

Next, we distinguish the following subcases.

Subcase 1.1 ( $R$ is not a polynomial). Suppose that $a_{0}$ is a pole of $R$ with multiplicity $m$. By (62) and the fact that $c \neq 1$ and $a_{0}$ is a pole of $\phi$ with multiplicity $m+k-1$, it contradicts (68).

Subcase 1.2 ( $R$ is nonconstant polynomial). From (62), $R_{1}$ is a polynomial. By simple calculation, we get $\operatorname{deg} R_{1}=$
$\operatorname{deg}\left(R\left(Q^{\prime}\right)^{k-1}\right)$. Combining with (68), we deduce $\operatorname{deg} R=$ $\operatorname{deg} R_{1}=\operatorname{deg}\left(R\left(Q^{\prime}\right)^{k-1}\right)=\operatorname{deg} R+\operatorname{deg}\left(Q^{\prime}\right)^{k-1}$. So $Q=m z+n$ and $\rho(\alpha)=1$. Here $m$ is a nonzero constant, and $n$ is a constant.

By integrating (59), we have

$$
f(z)=\lambda e^{c z}+A(z) e^{m z},
$$

where $\lambda$ is a nonzero constant and $A$ is a polynomial.

So $\rho(f) \leq 1=\rho(\alpha)$, a contradiction.

Subcase 1.3 ( $R$ is a constant). Set $R=a$. So $\alpha=a e^{Q}$. As subcase 1.2, we also get $Q(z)=m z+n$. Here $m$ is a nonzero constant, and $n$ is a constant. Integrating (59) yields

$$
f(z)=\left[a(1-c) \int e^{\mathrm{Q}(z)-c z} d z+c_{0}\right] e^{c z},
$$

where $\mathcal{c}_{0}$ is a constant.

If $m=c$, by calculation, $f(z)=\left(a b(1-c) z+c_{0}^{*}\right) e^{c z}$ (here $b=e^{n}$ and $c_{0}^{*}$ is an arbitrary constant), and $\rho(f)=\rho(\alpha)=1$, a contradiction.

So $m \neq c$, by calculation; $f(z)=a b(1-c)(1 /(m-c)) e^{m z}+$ $c_{0}^{*} e^{c z}$ (here $b=e^{n}$ and $c_{0}^{*}$ is an arbitrary constant), and $\rho(f) \leq$ $1=\rho(\alpha)$, a contradiction.

Case 2. If $Q$ is a constant, because $f$ is a transcendental meromorphic function, obviously the condition $\rho(\alpha)<\rho(f)$ and $\alpha \neq \alpha^{\prime}$ hold.

So Theorem 13 turns into Theorem 2, and the results of Theorem 2 still hold.

The proof is completed.

\section{Competing Interests}

The authors declare that they have no competing interests.

\section{Acknowledgments}

This work was supported by the NSF of China (11271090), Innovation Program of Shanghai Municipal Education Commission (14YZ164), Projects 13XKJC01 from the Leading Academic Discipline Project of Shanghai Dianji University, NSF of Guangdong Province (2015A030313346 and S2012010010121), Young Talents in Educational Commission of Guangdong Province (2015KQNCX116), National Natural Science Foundation of Guangdong Province (2016A030310257), and Shanxi Scholarship Council of China (no. 2013-045).

\section{References}

[1] H. X. Yi and C. C. Yang, Uniqueness Theory of Meromorphic Functions, Science Press, Beijing, China, 1995.

[2] W. Bergweiler, "On a theorem of gol'dberg concerning meromorphic solutions of algebraic differential equations," Complex Variables, Theory and Application, vol. 37, no. 1-4, pp. 93-96, 1998. 
[3] J. L. Schiff, Normal Families, Universitext, Springer, Berlin, Germany, 1993.

[4] W. J. Yuan, B. Xiao, and J. J. Zhang, "The general result of Gol'dberg's theorem concerning the growth of meromorphic solutions of algebraic differential equations," Computers \& Mathematics with Applications, vol. 58, no. 9, pp. 1788-1791, 2009.

[5] R. M. Gu, Z. R. Li, and W. J. Yuan, "The growth of entire solutions of some algebraic differential equations," Georgian Mathematical Journal, vol. 18, no. 3, pp. 489-495, 2011.

[6] R. Halburd and R. Korhonen, "Value distribution and linear operators," Proceedings of the Edinburgh Mathematical Society: Series 2, vol. 57, no. 2, pp. 493-504, 2014.

[7] R. Korhonen, "An extension of Picard's theorem for meromorphic functions of small hyper-order," Journal of Mathematical Analysis and Applications, vol. 357, no. 1, pp. 244-253, 2009.

[8] F. Lü, J. Xu, and A. Chen, "Entire functions sharing polynomials with their first derivatives," Archiv der Mathematik, vol. 92, no. 6, pp. 593-601, 2009.

[9] G. Zhang and J. Wang, "The infinite growth of solutions of complex differential equations of which coefficient with dynamical property," Taiwanese Journal of Mathematics, vol. 18, no. 4, pp. 1063-1069, 2014.

[10] G. Jank, E. Mues, and L. Volkmann, "Meromorphe Funktionen, die mit ihrer ersten und zweiten Ableitung einen endlichen Wert teilen," Complex Variables. Theory and Application, vol. 6, no. 1, pp. 51-71, 1986.

[11] J.-P. Wang, "Entire functions that share a polynomial with their derivatives," Journal of Mathematical Analysis and Applications, vol. 320, no. 2, pp. 703-717, 2006.

[12] F. Lü and H. X. Yi, "On the uniqueness problems of meromorphic functions and their linear differential polynomials," Journal of Mathematical Analysis and Applications, vol. 362, no. 2, pp. 301-312, 2010.

[13] S. Stevich, "Boundedness and compactness of an integral operator in a mixed norm space on the polydisk," Sibirskî Matematicheskîi Zhurnal, vol. 48, no. 3, pp. 694-706, 2007.

[14] S. Li and S. Stevich, "Riemann-Stieltjes-type integral operators on the unit ball in $C^{n}$," Complex Variables and Elliptic Equations, vol. 52, no. 6, pp. 495-517, 2007.

[15] X. C. Pang and L. Zalcman, "Normal families and shared values," The Bulletin of the London Mathematical Society, vol. 32, no. 3, pp. 325-331, 2000.

[16] F. Lü and J. Qi, "A note on the hyper-order of entire functions," Bulletin of the Korean Mathematical Society, vol. 50, no. 4, pp. 1209-1219, 2013.

[17] J. M. Chang, M. L. Fang, and L. Zalcman, "Normal families of holomphic functions," Illinois Journal of Mathematics, vol. 48, pp. 319-337, 2004.

[18] J. M. Chang and Y. Z. Zhu, "Entire functions that share a small function with their derivatives," Journal of Mathematical Analysis and Applications, vol. 351, no. 1, pp. 491-496, 2009.

[19] J. Grahl and C. Meng, "Entire functions sharing a polynomial with their derivatives and normal families," Analysis, vol. 28, no. 1, pp. 51-61, 2008.

[20] P. Yang and S. Nevo, "Derivatives of meromorphic functions with multiple zeros and elliptic functions," Acta Mathematica Sinica, vol. 29, no. 7, pp. 1257-1278, 2013.

[21] P. Yang, "Problems on $\left(f^{2}\right)^{(k)}$," Journal of Mathematical Analysis and Applications, vol. 335, no. 2, pp. 902-906, 2007. 


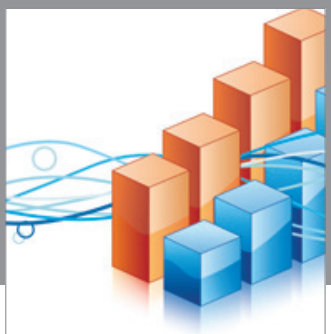

Advances in

Operations Research

vatem alat4

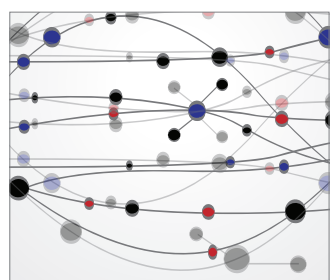

\section{The Scientific} World Journal
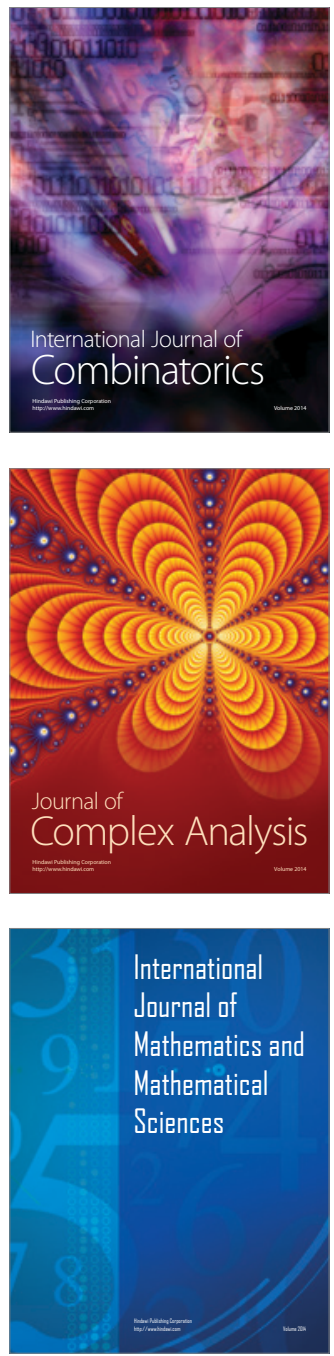
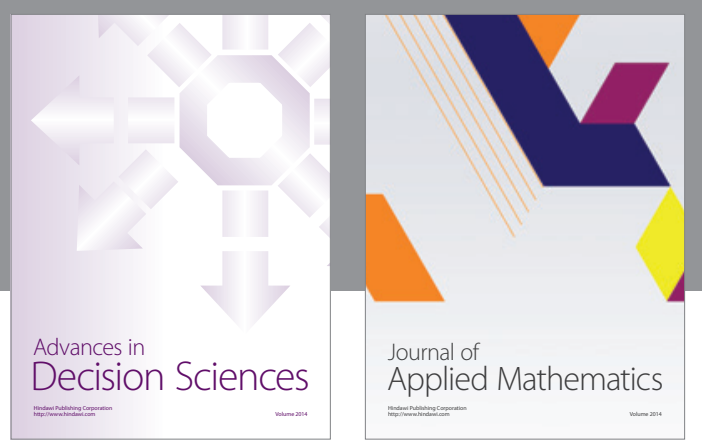

Algebra

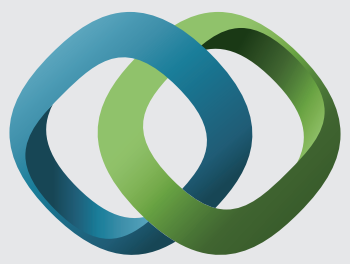

\section{Hindawi}

Submit your manuscripts at

http://www.hindawi.com
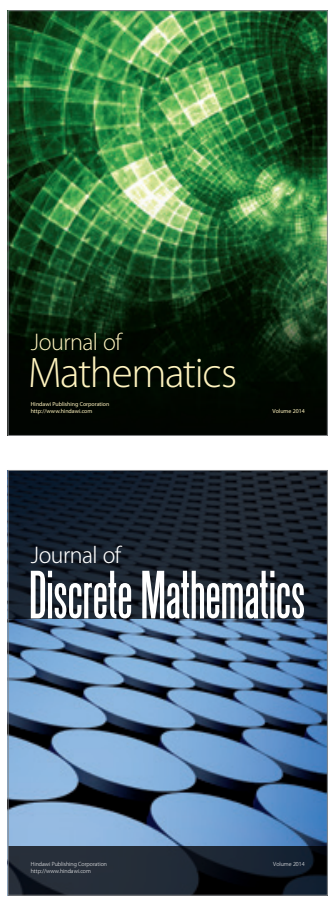

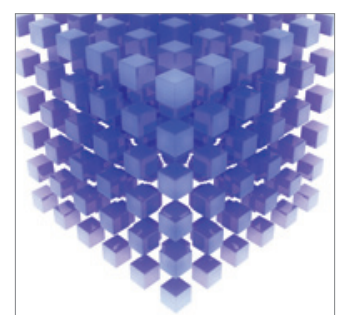

Mathematical Problems in Engineering
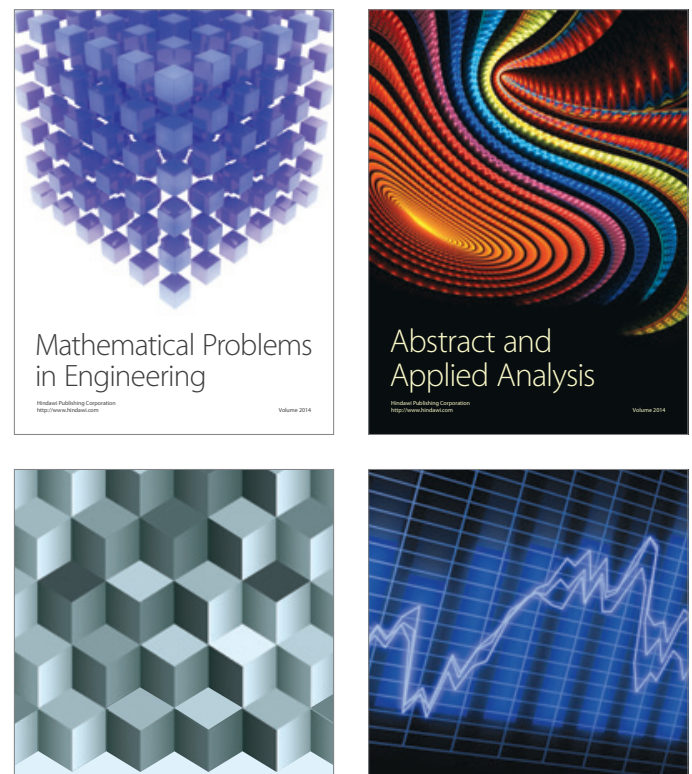

Journal of

Function Spaces

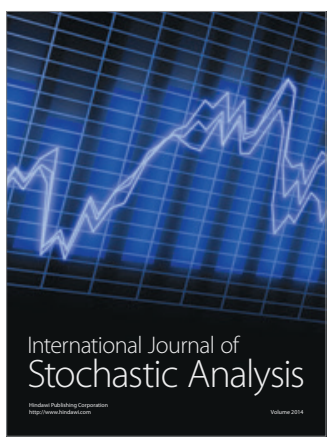

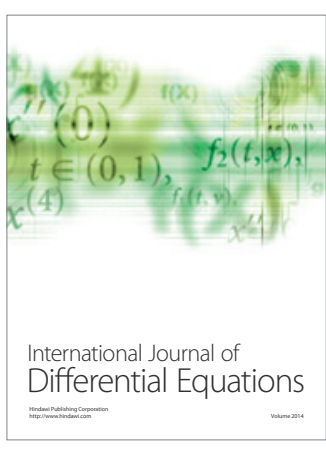
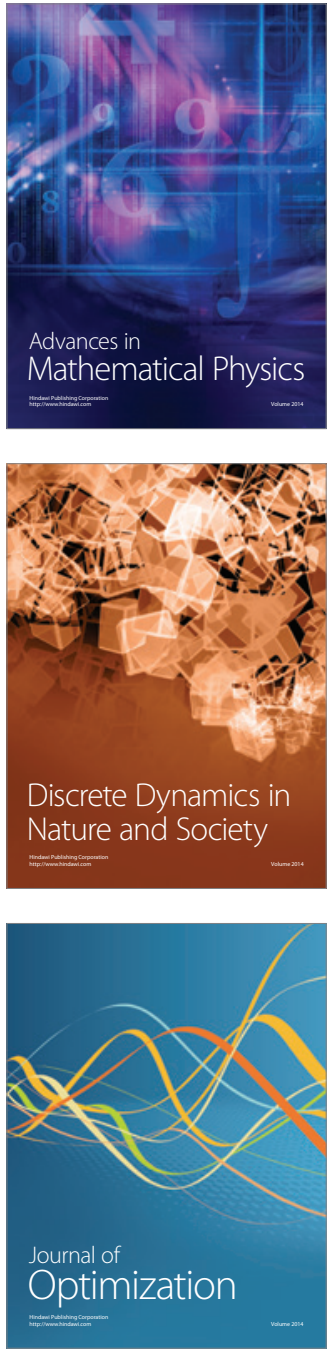